\title{
CREENCIAS Y PRÁCTICAS DE AUTOEVALUACIÓN DEL APRENDIZAJE VIGENTES EN LOS PROCESOS DE FORMACIÓN PROFESIONAL DESARROLLADOS EN EL CIDE DURANTE EL 2007
}

\author{
Sandra Ovares Barquero ${ }^{l}$ \\ Académica de la División de Educación Rural, CIDE-Universidad Nacional \\ Heredia, Costa Rica \\ Susana Ruiz, Guevara ${ }^{2}$ \\ Académica de la División de Educación Básica, CIDE-Universidad Nacional \\ Heredia, Costa Rica \\ Irma Zúñiga León ${ }^{3}$ \\ Decana del Centro de Investigación y Docencia en Educación, CIDE-Universidad Nacional \\ Heredia, Costa Rica
}

Recibido 18 de enero 2008• Aprobado 12 de marzo 2008

\begin{abstract}
Resumen: El presente artículo identifica creencias y prácticas de autoevaluación del aprendizaje vigentes en los procesos de formación profesional desarrollados en el CIDE durante el 2007, para lo cual se desarrolló un trabajo de investigación con dos grupos de informantes conformados por académicos del CIDE que, durante la década de los años 90 participaron en experiencias de autoevaluación de los aprendizajes como docentes o como estudiantes.

Las prácticas actuales que realizan por los académicos informantes en materia de autoevaluación de los aprendizajes en el CIDE, evidencian que ellos conceden gran importancia a este proceso en los cursos que imparten, y concuerdan en la necesidad de implementarlo de manera concreta, por cuanto las experiencias se convierten en iniciativas aisladas que no se enmarcan dentro de alguna política, lineamiento u orientación planteada por la comunidad CIDE.

Las creencias de los participantes con respecto a la posibilidad de aplicar procesos de autoevaluación de los aprendizajes en la formación profesional que desarrolla el CIDE, ponen de manifiesto la necesidad de capacitar al grupo de docentes y estudiantes en esta materia, y además, crear más espacios para la participación de éstos últimos. Por otra parte, los académicos creen que la autoevaluación de los aprendizajes es una estrategia para el desarrollo de procesos metacognitivos y reflexivos, lo que se considera de medular importancia en la formación profesional a cargo del Centro.
\end{abstract}

Palabras clave: Autoevaluación, aprendizaje, creencias, autorregulación

\begin{abstract}
This article identifies beliefs and practices of self-learning existing processes developed in the CIDE during 2007. It is based on a research project with two groups of informants both academics from CIDE, that during the decade of the 90 participated in the experience of self-learning, some of themas teachers and other as students.
\end{abstract}

Académica de la División de Educación Rural del CIDE, de la Universidad Nacional, Heredia, Costa Rica. Actualmente es la subdirectora. Académica de la División de Educación Básica del CIDE, de la Universidad Nacional, Heredia, Costa Rica. Doctora en Educación.

Decana del Centro de Investigación y Docencia en Educación (CIDE), de la Universidad Nacional, Heredia, Costa Rica. Catedrática de la Universidad Nacional. Máster en Desarrollo del Niño y Relaciones Familiares. 
Current practices being carried out by academics in the field of self-reporting of apprenticeships in the CIDE, show that they attach great importance to this process in the lesson and agree on the necessity to implement this process in concrete terms, that because, experiences become isolated initiatives that do not fall within any policy, guideline or orientation openly raised by the community CIDE.

The beliefs of the participants with regard to the possibility of implementing self-evaluation processes of learning in the training that develops the CIDE, highlight the need for scholars to train group of academics and students in this field and also create more spaces for student involvement. Moreover, scholars believe that the self-assessment of learning is a strategy for developing metacognitive processes and reflective, which is considered a core importance in the training provided by the Centre.

Key words : Education, experience of self-learning.

\section{INTRODUCCIÓN ¿DE DÓNDE PARTIMOS?}

Los procesos de autoevaluación del aprendizaje objeto de análisis de esta investigación, tienen su origen en los esfuerzos de innovación realizados en el Centro de Investigación y Docencia en Educación (CIDE) a finales de los años 80 y durante los años 90. Según Salas (s.f.), la necesidad de la autoevaluación se sustentaba en la idea de procurar una transformación de la educación hacia un proceso formativo. Con apoyo en Guba y Lincoln (1989), la autora en mención indica que los académicos universitarios manejan un sistema de creencias respecto de lo que significa ser docente, el cual, guía sus acciones y concepciones respecto a la evaluación y enfatiza que la explicitación de dicho sistema promueve el análisis crítico y la transformación de los procesos de enseñanza y aprendizaje. De ahí que el objetivo del presente trabajo consiste en identificar las creencias y prácticas de autoevaluación del aprendizaje vigentes en los procesos de formación profesional desarrollados en el CIDE durante el 2007.

\section{ALGUNAS IDEAS EN RELACIÓN CON LOS PROCESOS DE EVALUACIÓN Y LAS CREENCIAS}

\section{Aprendizajes derivados de la experiencia de evaluación del CIDE}

En cuanto a los significados de la autoevaluación y la coevaluación en la formación de maestros, para un grupo de estudiantes y docentes de la División de Educación Rural en la Universidad Nacional, las expresiones de los participantes en el estudio evidenciaron "...la construcción de significados derivados del discurso del deber ser dentro de una perspectiva de la educación centrada en resultados" (Salas, s.f., p. 25). Los profesores enfatizaron en los productos tangibles del aprendizaje y en los puntos para aprobar el curso; también en problemas diversos asociados con los procesos de autoevaluación, tales como: trabajo en grupos, orientación del profesor, forma de calificar, problemas familiares, falta de claridad en el proceso, y divorcio entre lo que piensa y quiere el profesor y lo que sucede con los estudiantes. Se destaca la carencia del diálogo para ayudar a tener claridad de lo que se espera y su importancia para la negociación franca, que ayuda a replantear los sistemas de creencias y a propiciar las transformaciones de conceptos y actitudes de los estudiantes y docentes. La autora señala la relevancia de que los académicos vean el potencial de los estudiantes, y que estos se vean a sí mismos como capaces de tomar decisiones responsablemente. Agrega que un nuevo sistema de creencias se construye en espacios de reflexión y de negociación, en la creación 
de propósitos comunes con la claridad de las tareas y responsabilidades específicas, no como una relación antagónica, sino en procura de la cooperación y de crecimiento compartido.

\section{Procesos de autoevaluación centrados en el aprendizaje}

En el contexto de la participación del CIDE en el Proyecto Evaluación Orientada al Aprendizaje Universitario (EVALCAU), es oportuno explorar las creencias y prácticas de autoevaluación del aprendizaje vigentes en los procesos de formación profesional desarrollados en el CIDE durante el 2007, con la claridad de que el enfoque por competencias que enmarca a EVALCAU no ha sido asumido en el Centro, lo cual representa una limitación para efectos de este estudio.

El reconocimiento de que la profesión docente se desarrolla en un espacio cultural que requiere que el estudiante interprete e intervenga en el mundo, implica trasformaciones en los procesos evaluativos. Diversas posiciones en torno a la evaluación, parten de que el control sobre el conocimiento y el aprendizaje debe trasladarse del educador al educando, quien adquiere un papel activo y más responsable; destacan la necesidad de que el estudiante reciba una oportuna realimentación sobre su desempeño a fin de mejorarlo, razón por la cual los criterios y las evidencias que permiten reconocer un buen trabajo deben ser claros y negociados con los actores de los procesos.

En otras palabras, los estudiantes, empleadores, padres de familia, académicos y la sociedad en general, son parte interesada en estos procesos. Esta perspectiva de la evaluación lleva consigo una discusión con los estudiantes en relación con cuánto les va beneficiar, los requerimientos establecidos y cómo será juzgada su actuación. Reconoce que los procesos evaluativos son medios para que los profesores aprendan a partir de la evaluación de sus alumnos (Trillo, 2008; Brown y Glasner, 2007). Desde esta perspectiva, la autoevaluación de los aprendizajes prepara para el desempeño laboral a largo plazo, desarrollando capacidades para generar procesos de mejoramiento permanentes en el ejercicio profesional; es decir, para adquirir habilidades personales de evaluación, de autovaloración crítica, de escuchar y responder a la realimentación, implica además, un componente reflexivo en torno a discusiones del valor añadido y el progreso, y se relaciona con el desarrollo del aprendizaje duradero.

La autoevaluación se emplea "...cada vez más de distintas formas para evaluar habilidades, conocimientos y competencia. Pero la capacidad de autoevaluar efectivamente no tiene lugar por sí misma. Los estudiantes necesitan una práctica sistemática para juzgar su propio trabajo y obtener un feedback sobre su capacidad de hacerlo" (Brew, en Brown y Glasner, 2007, p. 180). En resumen, desde la perspectiva del enfoque por competencias, y en particular de la evaluación centrada en el aprendizaje, es necesario que los académicos tomen conciencia de la forma en que están evaluando y del efecto profundo que la evaluación tiene sobre la vida de otros.

\section{Creencias}

Las creencias definen o bien determinan las actitudes de las personas, esto por cuanto se convierten en las orientaciones generales que el individuo construye en su interacción con el mundo. Las creencias se refieren a los principios que fundamentan la comprensión de los individuos o 
grupos sociales en relación con los fenómenos, y se definen como los referentes que “...dan cuenta del conjunto de las expresiones verbales y conductuales de los actores estudiados" (Muchelli, citado por Ruiz, 2006, p. 66 ).

Según Ortega y Gasset, las creencias forman parte de la vida, e inclusive, se manifiestan como la base sobre la cual las personas manejan esa vida. Toda conducta, incluso la intelectual, depende del sistema de creencias, razón por la cual no se tiene conciencia expresa de ellas; no se piensan, sino que actúan latentes, “...como implicaciones de cuanto expresamente hacemos o pensamos" (citado por Ruiz, 2006, p. 65).

Desde la perspectiva de Mansilla, las creencias originalmente eran ideas construidas por el ser humano para enfrentar el mundo; con el tiempo, se consolidaron y convirtieron en creencias. La creencia es, para Hume como esa idea fuerte y vivaz, derivada de impresiones que se dan en un momento determinado y que se relacionan directamente con esa idea. Tal y como lo indica Quintana, las creencias son, ante todo, personales y sociales. En el primer caso son construidas por el individuo, y por lo tanto, le pertenecen; sin embargo, ello no quiere decir que durante el proceso de la vida, el ser humano no pueda cambiarlas por otras. Indica el autor que se consideran un hecho social, en la medida en que el ser humano se desenvuelve en grupos sociales; las construcciones, a manera de creencias, están permeadas de ese contacto con el medio social, y por supuesto, puede afirmarse que lo mismo ocurre a la inversa: los grupos sociales se ven influidos por las creencias personales de los individuos que los conforman. En ese sentido, Sánchez establece que las creencias se consideran un producto social y personal, en tanto en muchos casos, son subconscientes, influenciando la autopercepción, la de los demás y de las cosas y situaciones que nos rodean (citados por Ruiz, 2006).

Por su parte, Mansilla indica que la creencia como producto de una idea surge en el plano individual, comprobándose en múltiples ocasiones hasta introducirse en el campo social que es en definitiva, donde se consolida. Este aspecto individual y colectivo de la creencia, es también asociado por Bueno a la realidad misma, que se convierte en el marco de referencia que utiliza el individuo para materializar sus creencias. Desde otra perspectiva, Chajet (2000) sugiere que las creencias pueden definirse como ideas que se acompañan de sentimientos de certeza, por cuanto con ellas se percibe el mundo de manera muy particular, son los anteojos que facilitan la percepción de problemas y soluciones y dan seguridad. Sánchez sugiere que por medio de las creencias y valores, las personas dan significado y coherencia al modelo del mundo con el cual se establece una vinculación directa (citados por Ruiz, 2006).

En síntesis, las comparaciones anteriores permiten sugerir que el plano afectivo y el cognitivo se evidencian como los ámbitos en los cuales se originan y rigen las creencias; asimismo, éstos tienen su referente en la realidad y permiten ser interpretadas. Las definiciones anteriores confirman un patrón de desarrollo asociado directamente con los planos individual y social, opciones válidas de donde emanan las creencias. Toda creencia nace como una respuesta del individuo ante la realidad, gestándose, de esta manera, en el plano individual. Sin embargo, es en el contacto con el mundo exterior cuando se evidencia la necesidad de una serie de comprobaciones que exige la consolidación de la creencia misma, lo que afecta no sólo el comportamiento de la persona como ser individual del grupo, sino también, del grupo que pertenece a esa realidad. Las creencias provocan que la persona se enfrente al mundo con una serie de ideas consolidadas que en cierta medida, no sólo determinan los intercambios con ese mundo, sino que, además, dan una realidad ya construida en la mente del individuo. Por eso, los cambios que se puedan generar acerca de su significado resultan una tarea verdaderamente difícil. 


\section{EL CAMINO COMPARTIDO POR LAS INVESTIGADORAS Y LOS INFORMANTES}

Con el fin de explorar las creencias y prácticas de autoevaluación del aprendizaje vigentes en los procesos de formación profesional desarrollados en el CIDE durante el 2007, se conformó un grupo focal y se aplicó un cuestionario a informantes claves de todas las unidades académicas del CIDE, con diferentes años de experiencia. Se pretendía obtener, con ambos grupos de informantes, una representación del equipo de académicos que participó a finales de los años 80 y durante los años 90 en la experiencia que desarrolló el CIDE en torno a procesos de autoevaluación, coevaluación y evaluación unidireccional como parte de la formación profesional ofrecida (G1). Se incorporaron además profesionales que fueron estudiantes durante ese periodo y que ahora son académicos en ejercicio en el Centro (G2).

El primer grupo estuvo constituido por seis informantes (G1) y el segundo (G2) por un total de catorce graduados del CIDE que actualmente son académicos. Cabe aclarar que el menor número de participantes en el primer grupo, se debe a que la mayoría de funcionarios que participaron en la experiencia de los años 80 y 90 se encuentran jubilados.

En ambos grupos de informantes (G1 y G2) se aplicó un cuestionario en forma individual, y luego, se procedió a una etapa de socialización durante la cual las investigadoras tomaron nota de las creencias expresadas por los participantes. Después de recopilada la información, se transcribieron las respuestas textualmente y se pasó a la construcción de categorías por separado y según el grupo de informantes. Estas categorías emergentes fueron contrastadas con la literatura y sirvieron de base para los principales hallazgos y recomendaciones derivadas del estudio.

\section{ALGUNAS REFLEXIONES EN TORNO A LOS RESULTADOS}

Los principales hallazgos se presentan según el grupo de informantes (G1 o G2) por categoría y se respaldan en expresiones textuales de los participantes.

Los datos relacionados con los aprendizajes obtenidos por los informantes del G1 coinciden con los expresados por el G2, en una de las funciones que cumple la autoevaluación y que se relaciona con la posibilidad de identificar fortalezas y debilidades en el desempeño académico de los estudiantes, tal y como se indica en las siguientes frases textuales "Cuando hay autoevaluación se constituye en un espacio que permite detectar las fortalezas y las debilidades, tanto en el desarrollo del curso, como en la interacción con los alumnos" (participante del G1), "La objetividad y reconocimiento de mis fortalezas y debilidades, reconocimiento de factores que inciden en los procesos de enseñanza y aprendizaje" (participante del G2).

Por otra parte, el G1 evidencia en sus respuestas que los procesos de autoevaluación requieren de una preparación tanto del estudiante como de los profesores, razón por la cual, insisten en la necesidad de un "...cambio de paradigma”, y que "faltó inducción y formación para manejar los procesos de autoevaluación tanto para estudiantes como para académicos". Por otro lado, aseguran que en el proceso "... debe ser gradual la asignación de cuotas para la autoevaluación”. Esta posición del G1 no coincide con respuestas del G2, ya que este último grupo expresa los aprendizajes en términos de habilidades personales y profesionales, según se indica en las siguientes frases "Capacidad crítica y analítica de mis experiencias", "El auto evaluación es una experiencia que me permite conocer con mayor profundidad lo que necesitan desean las personas con quienes comparto mi trabajo"; no así en función de la manera en que 
debe operar el proceso, o bien, de los requerimientos institucionales que se ocupan para que éste sea operacionalizado.

Otro aprendizaje obtenido por el G1, se refiere a que la autoevaluación de los aprendizajes generó aportes importantes para el estudiante, lo anterior se explica por el hecho de que la mayoría de las respuestas (4) de este grupo se orientan a considerar dicho proceso como una experiencia que le permite al grupo de discentes aprender, mejorar y valorar sus aportes y desempeños. Lo anterior contrasta con un grupo reducido de respuestas (2), en las cuales, los profesores del G1 dijeron haber aprendido algo desde su función personal y no desde lo que los estudiantes aprendieron. Esta situación pone de manifiesto que se requiere una interiorización mayor del profesorado con respecto al valor de la autoevaluación como práctica reflexiva de su labor docente.

El último aprendizaje que surge como categoría recurrente en las respuestas del G1, se relaciona con la utilidad de crear instrumentos como herramientas para fortalecer la autoevaluación de los aprendizajes.

Los datos aportados por los participantes del G2 en términos de sus aprendizajes, pueden organizarse en 4 grandes categorías: la primera de ellas, y más recurrente, se refiere a la necesidad de autocrítica para determinar fortalezas y debilidades, como medio para la mejora continua; en segundo término se cita la reflexión acerca de los procesos de enseñanza y aprendizaje desarrollada por los participantes. Como tercer aprendizaje, se establecen la valoración del desempeño en el curso y la identificación de los compromisos adquiridos; y como cuarta categoría, se señala la responsabilidad en el desarrollo del proceso de aprendizaje, por parte de los estudiantes, así como de sus progresos y compromisos. Cada una de estas categorías se detalla en los siguientes párrafos.

En el caso de las categorías relacionadas con procesos de autocrítica y de reflexión, los participantes del G2 señalan que con estos procesos lograron identificar sus limitaciones de formación, así como puntualizar las fortalezas y las debilidades en su desempeño como estudiantes. Por otra parte, los informantes del G2 señalan que la reflexión, en el marco de la autoevaluación, les permitió analizar la manera en que construyeron conocimientos y el reconocimiento de sus estilos de aprendizaje, lo cual evidencia el potencial de este proceso como una estrategia que favorece la metacognición.

En lo relativo a la valoración del desempeño, los participantes del G2 indican que la autoevaluación les ofreció la oportunidad de profundizar en sus trabajos e identificar el grado de cumplimiento de los compromisos adquiridos a lo largo del curso; por lo tanto, la autoevaluación se convierte en una estrategia que hace del estudiante un partícipe de sus procesos, y también de sus responsabilidades en los diferentes cursos.

En la cuarta categoría, titulada: "La responsabilidad en el desarrollo del proceso de aprendizaje por parte de los estudiantes, así como de sus progresos y sus compromiso"; el G2 indica en sus respuestas que, al ser un proceso formativo, se exige independencia para el aprendizaje y asumir responsabilidades, lo cual hace de la autoevaluación una herramienta que potencia el aprendizaje autónomo.

A manera de síntesis, es necesario indicar que los grupos G1 y G2 experimentan e interpretan la autoevaluación desde sus posturas como enseñantes y estudiantes, razón por la cual, el alcance del aprendizaje va desde una función estructural del proceso (enseñantes) hasta funciones que potencian las habilidades personales y profesionales. Estos datos son interesantes, especialmente, si se comparan con datos históricos de la experiencia de autoevaluación del CIDE, que no 
contemplaron el desarrollo de competencias con repercusión en el futuro ámbito profesional de los estudiantes.

Después de un recorrido por los aprendizajes construidos en el G1 y G2, se les consultó acerca de experiencias de autoevaluación implementadas en los cursos que imparten. Los datos indican que en el G1, 5 profesores utilizan la autoevaluación tanto con propósitos formativos como para que el estudiante valore su trabajo y le asigne una nota numérica, utilizando para ello, una escala tipo Lickert de cinco puntos, con afirmaciones sobre aprendizajes, esfuerzos y logros.

También se les preguntó a los participantes de ambos grupos, el porqué de dichas experiencias; las respuestas del G1 fueron muy variadas y se evidencian en la siguientes frases: "Es importante hacerlo para retroalimentar todas las fortalezas, minimizando las debilidades para detectar cuáles son mis necesidades de formación", "Para generar procesos metacognitivos y en el ámbito sumativo, la utilizo para el análisis de los resultados de las pruebas escritas con el grupo de I y II Ciclos"; "Lo hago porque creo que no todos y todas dan lo mismo en esfuerzo y compromiso, no se trata de quién llega más lejos, sino quien tiene más logro desde su nivel de entrada a su nivel de salida"; "Con el objetivo de que se reconozcan debilidades y avances"; "Para que cada participante descubra por sí mismo el grado de compromiso, pertinencia y de investigación que dedicó al proceso y a su aprendizaje".

Las frases expuestas evidencian que el G1 reconoce abiertamente que la autoevaluación debe tener un por qué al momento de ser aplicada, lo cual coincide con la literatura, especialmente porque implica un proceso de reflexión en sus actores. Por otra parte, si bien es cierto las respuestas del G1 son variadas, las razones de peso por las cuales se implementa la autoevaluación, se dirigen a que el estudiante valore su trabajo e identifique sus fortalezas y debilidades.

En el Cuadro 1 se resumen las experiencias de autoevaluación que aplican los participantes del grupo G2, y las razones por las cuales lo hacen.

Ese Cuadro evidencia que 12 participantes del G2 sí aplican experiencias de autoevaluación en los cursos que imparten, y pueden describirlas; mientras que 2 no lo hacen. Además, 7 personas identifican las razones que justifican la aplicación de sus experiencias, mientras que las restantes 5 no indican el por qué, lo cual pone de manifiesto la necesidad de reflexionar sobre la importancia de la autoevaluación como práctica pedagógica. Por otra parte, es evidente que las descripciones de las experiencias sugieren la necesidad de fortalecer su sistematización y evaluación.

Otro dato interesante se refiere a la intención de la autoevaluación, la cual se orienta en la mayoría de las ocasiones, a una dimensión formativa, no sumativa; esta situación pone de manifiesto que existe una creencia implícita entre los académicos de que lo sumativo y formativo no son complementarios en el proceso de autoevaluación de los aprendizajes de sus estudiantes.

Lo anterior contrasta con lo expuesto en la literatura, en la cual se expresa que favorece la autoevaluación de habilidades que se relacionan con la preparación del estudiante para asumir, en un futuro cercano, el rol de profesional que enfrenta situaciones de trabajo en equipo y valoración de los aportes de sus integrantes; por tanto, el estudiante debe autoevaluar su trabajo y asignarse valores para convertirlo en una acción con cierto grado de tangibilidad ante la mirada de otras personas.

La información del Cuadro 1 también evidencia que existe poca relación entre las experiencias que aplican los participantes del G2; es decir, no puede establecerse un patrón definido que se base en políticas o lineamientos sobre la autoevaluación en el CIDE; esto se debe a que el marco de evaluación de los aprendizajes de dicho Centro, no hace explícita la autoevaluación. 


\section{Cuadro 1}

Experiencias de auto evaluación que implementan los profesores del G2 y las razones que la justifican

\begin{tabular}{|c|c|c|c|}
\hline Profesor & $\begin{array}{l}\text { Aplica } \\
\text { Si/no }\end{array}$ & Descripción de la experiencia & ¿Por qué? \\
\hline 1 & Sí & $\begin{array}{l}\text { Entre trabajos que se les asignan a los } \\
\text { estudiantes, se establece un apartado } \\
\text { donde ellos tienen que autoevaluar su } \\
\text { desempeño. } \\
\text { Además, durante el 2006, se invitó a los } \\
\text { estudiantes a evaluar los trabajos de expo- } \\
\text { sición con un puntaje máximo de } 20 \text { pun- } \\
\text { tos; sin embargo, requirió de la mediación } \\
\text { del profesor, ya que los estudiantes no se } \\
\text { distribuyeron las notas equitativamente, de } \\
\text { acuerdo con el trabajo de cada uno. }\end{array}$ & $\begin{array}{l}\text { "Porque considero un ideal de la educación que cada } \\
\text { persona se desempeñe eficientemente sin que tengan } \\
\text { que existir controles. A los estudiantes, se les debe } \\
\text { formar en una cultura de la autoevaluación, donde cada } \\
\text { uno asuma su papel en la sociedad, sea congruente y } \\
\text { responsable ante el". }\end{array}$ \\
\hline 2 & Sí & $\begin{array}{l}\text { En el trabajo final de curso los estudiantes } \\
\text { deben realizar una reflexión de las obser- } \\
\text { vaciones y prácticas realizadas. }\end{array}$ & "Pienso que esto es más fructífero" \\
\hline 3 & Sí & $\begin{array}{l}\text { Se aplica una experiencia de toma de } \\
\text { conciencia, por parte de los estudiantes } \\
\text { y la profesora, del compromiso con la } \\
\text { formación, "...por consiguiente cada quien } \\
\text { es responsable de los aprendizajes y las } \\
\text { enseñanzas que genera y propone en el } \\
\text { aula y fuera de ella". }\end{array}$ & $\begin{array}{l}\text { "Lo hago porque deseo que las y los educandos me } \\
\text { ayuden a crear los mejores ambientes para la enseñanza } \\
\text { y el aprendizaje en el aula universitaria, para que vivan } \\
\text { una experiencia que enseña en su esencia-vivencia y no } \\
\text { solo desde el discurso teórico" }\end{array}$ \\
\hline 4 & Sí & $\begin{array}{l}\text { "Los estudiantes desarrollan sus portafo- } \\
\text { lios con reflexiones creativas y críticas ela- } \\
\text { boradas semanalmente. Cada dos semanas } \\
\text { se comparten los portafolios en dos cír- } \\
\text { culos concéntricos donde los estudiantes } \\
\text { y las estudiantes se ubican de frente. Las } \\
\text { personas ubicadas en el círculo interno } \\
\text { giran a la izquierda al cabo de unos } 5 \text { ó } \\
10 \text { minutos y comparten sus trabajos con } \\
\text { otros compañeros y compañeras. } \\
\text { Les proporciono una hoja para que, ade- } \\
\text { más del comentario oral que hacen de los } \\
\text { portafolios de sus compañeros y compañe- } \\
\text { ras, documenten una observación escrita. } \\
\text { Los estudiantes y las estudiantes autova- } \\
\text { loran su desempeño en el curso, con base } \\
\text { en unas rúbricas que se les ofrece. Estas } \\
\text { son congruentes con los elementos plan- } \\
\text { teados en la estrategia de evaluación del } \\
\text { programa del curso. Esta es una valoración } \\
\text { cualitativa que no afecta su nota final". }\end{array}$ & $\begin{array}{l}\text { "Porque esta forma de autovaloración en su portafolio, } \\
\text { les permite a ellos y ellas aprender, no sólo del docente, } \\
\text { sino de sus compañeros y compañeras, al co-valorar } \\
\text { sus trabajos, se estimula el crecimiento académico y } \\
\text { esfuerzo que se pone en el portafolio. Esto constituye } \\
\text { uno de los objetivos de la evaluación que es permitir } \\
\text { el mejoramiento. Además, el tiempo es maximizado al } \\
\text { estar todas las personas del curso hablando y compar- } \\
\text { tiendo al mismo tiempo. } \\
\text { Se utiliza como mecanismo de autorreflexión para el } \\
\text { mejoramiento personal y académico". }\end{array}$ \\
\hline
\end{tabular}




\begin{tabular}{|c|c|c|c|}
\hline Profesor & $\begin{array}{c}\text { Aplica } \\
\text { Si/no }\end{array}$ & Descripción de la experiencia & ¿Por qué? \\
\hline 5 & Sí & $\begin{array}{l}\text { Aplica preguntas generadoras que llevan } \\
\text { a la reflexión del proceso de aprendizaje, } \\
\text { como forma de que el estudiante autovalo- } \\
\text { re sus aprendizajes y esfuerzos invertidos. }\end{array}$ & No se indica \\
\hline 6 & & $\begin{array}{l}\text { Utiliza la auto y la co evaluación a lo largo } \\
\text { de las experiencias de aprendizaje que se } \\
\text { establecen en el curso; además, negocia } \\
\text { con las estudiantes los criterios valorativos } \\
\text { que se incluyen en las rúbricas que sirven } \\
\text { de guía. }\end{array}$ & No se indica \\
\hline 7 & Sí & $\begin{array}{l}\text { "Lo hago, usualmente, al final de los } \\
\text { cursos como un ejercicio que permita al } \\
\text { estudiante reflexionar sobre sus procesos } \\
\text { de aprendizaje". }\end{array}$ & $\begin{array}{l}\text { "Lo hago usualmente al final de los cursos como un } \\
\text { ejercicio que permita al estudiante reflexionar sobre sus } \\
\text { procesos de aprendizaje". }\end{array}$ \\
\hline 8 & Sí & $\begin{array}{l}\text { Durante el proceso de construcción de su } \\
\text { anteproyecto de graduación, trabaja conti- } \\
\text { nuamente con sus estudiantes, identifican- } \\
\text { do e introduciendo mejoras al documento. } \\
\text { Dichas mejoras se discuten, reflexionan } \\
\text { y se analizan en función de su viabilidad } \\
\text { para el proyecto en general. } \\
\text { Al final del proceso (dos ciclos lectivos), } \\
\text { se le pide a la estudiante que autoevalúe su } \\
\text { trabajo desde una óptica formativa. }\end{array}$ & $\begin{array}{l}\text { "Porque considero que esto les permite a las estudiantes } \\
\text { identificar sus debilidades y fortalezas tanto en térmi- } \\
\text { nos de los requerimientos propios del curso, como tam- } \\
\text { bién de su formación hasta el momento. Para fortalecer } \\
\text { habilidades relacionadas con una valoración constante } \\
\text { del trabajo que realizan las estudiantes, buscando el } \\
\text { fortalecimiento de una cultura de la evaluación profe- } \\
\text { sional constante". }\end{array}$ \\
\hline 9 & Sí & $\begin{array}{l}\text { Promueve que las y los estudiantes valoren } \\
\text { su trabajo en el curso, y además, el profesor } \\
\text { reflexiona acerca de las experiencias y logros } \\
\text { del curso para analizar su pertinencia. }\end{array}$ & $\begin{array}{l}\text { "Lo hago como un compromiso personal, a los y las } \\
\text { estudiantes les doy un guía para que me externen sus } \\
\text { criterios de manera libre y respetuosa". }\end{array}$ \\
\hline 10 & Sí & $\begin{array}{l}\text { "Si en el momento que realizan una tarea } \\
\text { se les pregunta si realmente pusieron todo } \\
\text { el empeño que podían haber puesto. } \\
\text { Se les pregunta la nota que consideran que } \\
\text { merecen ante un trabajo, les menciono que } \\
\text { hay dos opciones decir la verdad o mentir } \\
\text { por lo general responden muy bien y con } \\
\text { sinceridad". }\end{array}$ & No se indica \\
\hline 11 & Sí & $\begin{array}{l}\text { De manera informal, se invita a las estu- } \\
\text { diantes a reflexionar sobre la ejecución } \\
\text { de su proyecto (proyecto Pedagógico de } \\
\text { aula). }\end{array}$ & No se indica \\
\hline 12 & No & No aplica experiencia & No se indica \\
\hline
\end{tabular}




\begin{tabular}{|l|l|l|l|}
\hline Profesor & $\begin{array}{c}\text { Aplica } \\
\text { Si/no }\end{array}$ & \multicolumn{1}{|c|}{ Descripción de la experiencia } & ¿Por qué? \\
\hline 13 & Sí & $\begin{array}{l}\text { "Le hago saber a los estudiantes en sus } \\
\text { trabajos escritos y orales los aspectos } \\
\text { positivos y retos por considerar y esto es } \\
\text { respaldado por medio de hojas de cotejo } \\
\text { previamente establecidos con los estu- } \\
\text { diantes". }\end{array}$ & No se indica \\
\hline 14 & Sí & No aplica experiencia. & No lo aplica \\
\hline
\end{tabular}

Después de conocer lo que hacen los profesores del G1 y G2 en sus aulas, en relación con experiencias concretas de autoevaluación, se les consultó acerca de sus creencias con respecto a una aplicación de procesos de autoevaluación del aprendizaje, dentro de las actividades formativas que desarrolla el CIDE. Ante esta consulta, el total de participantes de ambos grupos (G1 y G2) insisten en la necesidad e importancia de impulsar un proyecto de este tipo para fortalecer los procesos de formación que lidera el CIDE; asimismo, en ambos grupos las respuestas coinciden con respecto a creencias sobre la implementación de la autoevaluación en los procesos de aprendizaje del CIDE:

Cuadro 2

Creencias de profesores (G1) y estudiantes-profesores (G2), en relación con la aplicación de procesos de auto evaluación del aprendizaje, en los procesos formativos desarrollados en el CIDE

\begin{tabular}{|c|c|c|}
\hline Descripción de la categoría & Frecuencia & $\%$ \\
\hline $\begin{array}{l}\text { Requiere capacitación y compromiso de parte de profesores y estudiantes: (Frases } \\
\text { textuales que ejemplifican la categoría): } \\
\text { "Retomar los procesos como una acción creativa, constructiva, no como un aspecto permisi- } \\
\text { vo", "Considero que sería muy valioso, pero son procesos delicados donde se debe preparar } \\
\text { tanto al estudiante como al profesor", "Falta capacitación en el tema", "Considero necesario } \\
\text { capacitación en el tema" "Creo que será posible y exitoso en la medida en que académicos, } \\
\text { académicas y estudiantes seamos capaces de interiorizar la importancia que tienen los } \\
\text { procesos de auto evaluación para el crecimiento personal y Professional. Se debe potenciar } \\
\text { estrategias con nuestros estudiantes", "Tomar grupos determinados y dar fuerte inducción } \\
\text { a los y las estudiantes". }\end{array}$ & 11 & 28,95 \\
\hline $\begin{array}{l}\text { Se debe implementar gradualmente y a lo largo del curso lectivo: (Frases textuales que } \\
\text { ejemplifican la categoría). } \\
\text { "Trabajar progresivamente por nivel", "Es un proceso gradual de convicción del porque } \\
\text { y para que se hace", "Se debe trabajar durante un tiempo prudencial y dar informes para } \\
\text { enriquecer el proceso", "Esto no debe dejarse para el final del curso, debe ser permanente } \\
\text { en todo el proceso y debe participar el docente como facilitador". }\end{array}$ & 7 & 18,42 \\
\hline
\end{tabular}




\begin{tabular}{|c|c|c|}
\hline Descripción de la categoría & Frecuencia & $\%$ \\
\hline $\begin{array}{l}\text { Genera destrezas relacionadas con la reflexión, el cambio y la metacognición: (Frases } \\
\text { textuales que ejemplifican la categoría): } \\
\text { "Me parece que se deben continuar realizando para que desarrollemos mayores destrezas } \\
\text { de reflexión, cambio y de gestión que al futuro nos permita crecer y adaptarnos al futuro, } \\
\text { "Me parece excelente la incorporación de la experiencia genera madurez, crecimiento y } \\
\text { capacidad de autocontrol", "Que se generen procesos metacognitivos", "Auto evaluación } \\
\text { sin reflexión, autocrítica, honestidad y un plan remedial de contingencia en caso de que el } \\
\text { alumno sea sincero en sus puntajes no tiene sentido". }\end{array}$ & 6 & $15,78 \%$ \\
\hline $\begin{array}{l}\text { Requiere de que se fijen criterios para su adecuado funcionamiento, alejados de la } \\
\text { valoración numérica: (Frases textuales que ejemplifican la categoría): } \\
\text { "Debían ir en función de que el estudiante se evalué frente a unos criterios que tienen que } \\
\text { ver con su perfil académico y con lo que se espera de el o ella para ese curso o nivel. Si es } \\
\text { muy subjetivo creo que no cumple su función", "Se debe negociar de previo los criterios a } \\
\text { tomar en cuenta, con los estudiantes", "El sistema numérico afecta mucho estos procesos, } \\
\text { se podría pensar en otras formas de dar los resultados de una promoción", "Promover el } \\
\text { diseño de instrumentos en el que participen los diferentes actores en los procesos de forma- } \\
\text { ción, validar los instrumentos y después de analizar la información, realizar la devolución } \\
\text { pertinente". }\end{array}$ & 5 & 13,15 \\
\hline $\begin{array}{l}\text { Requiere un proceso de madurez de estudiantes y profesores: (Frases textuales que } \\
\text { ejemplifican la categoría): } \\
\text { "Implica un procesote de madurez de los estudiantes, no se puede aplicar de la noche a } \\
\text { la mañana", "Los estudiantes requieren madurar de lo contrario los estudiantes pierden la } \\
\text { perspectiva de la función de la evaluación personal", "Se requiere madurez por parte de } \\
\text { estudiantes y profesores". }\end{array}$ & 3 & 7,90 \\
\hline $\begin{array}{l}\text { Permite la participación del estudiante en la evaluación: (Frases textuales que ejempli- } \\
\text { fican la categoría): } \\
\text { "Me parece que seria importante porque se critica que los y las estudiantes no saben aplicar } \\
\text { esta opción de evaluación pero si no se les da la oportunidad, la confianza, posiblemente no } \\
\text { lo van a aprender esta cultura tica requiere aprender a evaluar y mejorar en lo que sea nece- } \\
\text { sario, la evaluación enriquece si se aplica adecuadamente como autocrática", "Considero } \\
\text { puede ser importante, porque permite que el estudiante auto valore todo lo que es o no capaz } \\
\text { de dar de si mismo para alcanzar los objetivos de cada curso". }\end{array}$ & 3 & 7,90 \\
\hline $\begin{array}{l}\text { Otros: } \\
\text { Es una alternativa para conocer al estudiante. } \\
\text { Si se aplica la autoevaluación, debe aplicarse también la coevaluación dentro del proceso } \\
\text { para enriquecerlo mutuamente y este sea más objetivo e integral. } \\
\text { Creo que es muy importante porque podríamos estarnos enfrentando algunas variables que } \\
\text { desconocemos y que pueden resultar muy valiosas en los procesos de formación desarro- } \\
\text { llados en el CIDE. }\end{array}$ & 3 & 7,90 \\
\hline Total de respuestas & 38 & 100 \\
\hline
\end{tabular}

Los datos indicados en el cuadro anterior reflejan con claridad que la autoevaluación, como proceso, requiere preparar tanto a estudiantes como a profesores, así como gradualidad en su aplicación. Si bien es cierto, la literatura no señala requisitos para la aplicación de procesos autoevaluativos, identificar las creencias de los académicos es indispensable, ya que ellas actúan como necesidades; o bien, como directrices del desarrollo profesional en materia de autoevaluación. Otro 
dato importante, se refiere a la creencia de los profesores con respecto a lo positivo de aplicar la autoevalución en los procesos de aprendizaje que lidera el CIDE.

\section{CONCLUSIONES}

La autoevaluación influyó positivamente durante la formación recibida por académicos del G2 que imparten cursos en el CIDE, lo anterior debido a que les permitió desarrollar habilidades personales, intelectuales y profesionales que impactan su quehacer diario, y sobre todo, les facilita procesos de reflexión constante para la identificación de fortalezas y debilidades en su desempeño.

Los participantes del G1, que se definen como académicos que aplicaron la experiencia de autoevaluación, identifican sus aprendizajes en función de los requerimientos del proceso para ser aplicado, y de los aprendizajes que lograron los estudiantes; no obstante, no hacen referencia a procesos personales de cambio desde su visión como profesores, lo cual evidencia la necesidad de fortalecer en el académico los alcances personales y profesionales de aplicar experiencias de autoevaluación.

Los datos evidencian que la autoevaluación favoreció procesos metacognitivos y de aprendizaje autónomo en los participantes del G2, lo cual se considera muy importante en la formación de competencias requeridas para los profesionales del presente siglo.

La autoevaluación como experiencia de carácter formativo, se continúa aplicando en los procesos de aprendizaje del CIDE por académicos del G1 y G2; sin embargo, el impacto de este proceso no ha sido relevante en los aspectos sumativos, lo que evidencia que se requiere de un cambio de paradigma de la evaluación de los cursos, y también de la preparación de estudiantes y profesores.

Las experiencias de autoevaluación que continúan implementando los participantes del G1 y G2 en los procesos de enseñanza y aprendizaje que se desarrollan el CIDE, son muy variadas e influyen directamente en la valoración del desempeño de los estudiantes. La variedad de experiencias evidencia la necesidad de aplicar estos procesos en las dinámicas de aula; pero también, reflejan una ausencia de lineamientos claros acerca de la autoevaluación de los aprendizajes, lo anterior, debido a que la estrategia de autoevaluación seleccionada depende de la experiencia de cada profesor.

La totalidad de participantes del G1 y G2 confirman la necesidad e importancia de implementar procesos de autoevaluación de los aprendizajes en el CIDE.

Las creencias de los participantes del G1 y G2, sugieren que la autoevaluación tiene requerimientos y ofrece beneficios. Como parte de los requerimientos se habla de madurez. La literatura es clara en afirmar que la autoevaluación es un proceso que le pertenece en gran medida al estudiante, mientras que el profesor es un mediador; por eso, más que madurar, docentes y estudiantes requieren de lenguajes comunes, y las carreras del CIDE deben considerar las capacidades y saberes que desean favorecer en la formación del profesorado para construir propuestas más claras en relación con la autoevaluación de los aprendizajes.

El valor positivo que conceden los participantes del G1 y G2 a la autoevaluación, que se evidencia en sus creencias, permite identificar un campo fértil para iniciar procesos de reflexión y construcción de una evaluación de los aprendizajes centrada en los estudiantes, que repercuta a largo plazo en el desarrollo profesional de los educandos. 


\section{REFERENCIAS}

Brown, S. y Glasner, A. (2007). Evaluar en la Universidad Problemas y nuevos enfoques. Madrid: Narcea, S. A. de Ediciones.

Chajet, A. (2000). Creencias, esos anteojos tan particulares. En línea: www.teletel.com.ar/nplbaires/arties/ creen.htm.

Ruiz, S. (2006). Creencias que subyacen en la práctica docente de una maestra costarricense que atiende el ciclo de transición, en relación con el desarrollo integral de una niña y dos niños inmigrantes nicaragüenses que asisten al jardín infantil público Luces, ubicado en una zona de atención prioritaria de la provincia de Heredia: un estudio de casos. San José, Costa Rica. Tesis doctoral no publicada.

Salas, F. (s.f.). Los significados de la autoevaluación y la coevaluación en la formación de maestros, para un grupo de estudiantes y docentes de la División de Educación Rural en la Universidad Nacional. Universidad de Costa Rica, Sistema de Estudios de Posgrado, Facultad de Educación. Maestría en Evaluación Educativa.

Trillo, F. (2008). La evaluación del Aprendizaje de los Estudiantes en la Universidad. Antología del curso impartido del 27 de enero al 3 de febrero.w 